\title{
The Use of Additionally Trained Sonographers as Ultrasound Practitioners
}

\author{
Our First-Year Experience
}

Ronald O. Bude, MD, John P. Fatchett, AS, RDMS,

Tena A. Lechtanski, RT, RVT, RCDS, RDMS

\begin{abstract}
Objective. Two sonographers were trained to help manage an abrupt, permanent increase in the number of ultrasound examinations in our department. Called "ultrasound practitioners," they functioned as physician assistants and triaged 20 to 30 cases per day, allowing the cases to be batch read at a formal reading at day's end. We report our first-year experience with this program. Methods. Two sonographers with 10 and 30 years of experience, respectively, were trained to triage and dictate cases. Once trained, they triaged the cases of 20 to 30 patients per day. Reports were predictated with voice recognition technology. A radiologist was always readily available to provide support, and consultation with a radiologist was always obtained for the infrequent verbal reports that were requested. Reports from the practitioner were graded subjectively on a 4-point scale for the first year, according to the modification required at formal readout (A, no change; B, minor change not affecting patient care; $C$, moderate change not affecting care in a dramatic way; and $D$, major change markedly affecting care). Results. Practitioner 1 monitored the examinations of 2858 patients. The graded report results were as follows: A, 96.2\%; B, 3.5\%; C, 0.3\%; and D, 0.00\%. Practitioner 2 monitored the examinations of 2825 patients. The graded report results were as follows: $A, 96.1 \%$; $B, 3.6 \% ; C, 0.2 \%$; and $D, 0.00 \%$. There were no category D reports. Conclusions. The results far exceeded expectations, with a very low rate of category $B$ and $C$ reports and an absence of category D reports. The practitioners allowed the cases of 20 to 30 patients to be batch read by the existing radiologist staff at the end of the day. Key words: diagnostic radiology; efficacy study; observer performance; radiologists; radiology; socioeconomic issues; sonographer; ultrasound.
\end{abstract}

Abbreviations

ABR, American Board of Radiology; PACS, picturearchiving and communications system
Received September 14, 2005, from the Department of Radiology, University of Michigan Medical Center, Ann Arbor, Michigan USA. Revision requested October 3, 2005. Revised manuscript accepted for publication October 24, 2005.

Address correspondence to Ronald Bude, MD, B1D 502, Department of Radiology, University of Michigan Medical Center, 1500 E Medical Center Dr, Ann Arbor, MI 48109-0030 USA.

E-mail: ronbude@umich.edu

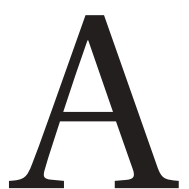

s early as the 1970s, radiologists advocated the use of additionally trained radiologic technologists. to assist in managing workflow. The duties of these assistants included screening plain films into positive and negative categories and performing, but not interpreting, fluoroscopic procedures. ${ }^{1} \mathrm{~A}$ bolder approach has been undertaken recently in England. In many hospitals, additionally trained radiologic technologists interpret and report, without supervision, musculoskeletal plain radiographs for the emergency department and for general practitioners. ${ }^{2-4}$

In addition, the concept of a skilled individual assisting a radiologist in the management and interpretation of ultrasound examinations is not new. Since the 1980s, 
A. Thomas Stavros, MD, and Beryl R. Benacerraf, $\mathrm{MD}$, each have had practices in which a single skilled sonographer has evolved into a sonographic "physician's assistant," whom they have termed "sonographer practitioner" and "advanced practice sonographer," ${ }^{6}$ respectively. In a recent editorial, Lockhart et $\mathrm{al}^{5}$ described their experience. They specifically established the position of sonographer practitioner to assist in the management of ultrasound examinations. Also, recognizing the need for such an individual to assist with ultrasound examinations, the Society of Diagnostic Medical Sonography has established criteria to define a position entitled "advanced practice sonographer."7 Reports of the experience with these ultrasound assistants have been in the form of editorials and have been narrative and descriptive in nature. ${ }^{5,6}$

Approximately 4 years ago, we encountered an abrupt increase in ultrasound examinations in our department, which created the need for an assistant to help manage our caseload. We created a position, which we entitled "ultrasound practitioner," to fit our local needs and recruited and trained 2 individuals to fill this new position. Data were kept on the first year of their performance with an eye to publication of the results. We report our experience with this program.

\section{Materials and Methods}

Two highly qualified and motivated sonographers with 10 and 30 years of experience, respectively, were interviewed and chosen to undertake the positions. They were trained and assessed before beginning their duties. A prospective study of their efficacy was performed for the first year of their work. Before the program commenced, our department chair reviewed the legal aspects with our hospital's legal counsel. It was concluded that there were no legal problems with our setup and that the program could be instituted.

Each candidate was given 1 week to observe case interpretation and dictation in the reading room and was given the same consideration as a medical student. This was followed by 4 weeks of on-the-job training, during which each candidate triaged and dictated reports under the close supervision of a radiologist. Training was scheduled so that the second practitioner began the 4 weeks of on-the-job training immediately after the first practitioner had finished training.
After training was completed, each candidate was subjectively judged to be competent by 2 radiologists in our department who had the most ultrasound examination experience ( 21 and 24 years). Each candidate was then assessed with an oral examination given by a radiologist who was an examiner in sonography for the American Board of Radiology (ABR). The examination consisted of 10 cases chosen to be of the same types and degrees of difficulty as those included in a recent $\mathrm{ABR}$ oral examination. Each examination had a 25-minute time limit. The practitioners were scored in the same manner as that used for the ABR examination, with scores of 68 to 72 , in integer values, for each case. At the end of the examination, the scores of the 10 cases were averaged; a passing score was a mean of 70 or higher, as used in the ABR examination.

After they passed the examination, the practitioners assumed their formal functions. They work an average of 10 hours per day for each shift to cover the day's off-site ultrasound cases as well as the after-hours' readout session (described later) and consequently work four 10-hour shifts per week (they consider this a substantial "perk"). Their pay is similar to that of physician assistants in our hospital.

The major responsibilities of each practitioner include the following: (1) Triage and evaluate offsite ultrasound studies for later review (the cases of approximately 20-30 outpatients per day; some patients had more than 1 ultrasound examination, eg, an abdomen and a pelvis ultrasound study, but we did not keep track of these as multiple examinations). These studies were performed off-site by sonographers and transferred via high-speed access to our in-house picturearchiving and communications system (PACS) (Acuson KinetDx; Siemens Medical Solutions, Mountain View, CA). Examinations consisted of both static images and video clips up to $10 \mathrm{sec}-$ onds long. Records regarding the case mix were not kept, but cases included abdominal, pelvic, thyroid, obstetric (all trimesters), carotid (but no other peripheral Doppler studies), and liver and renal Doppler examinations (including transplants of these organs). Pediatric patients were not included. (2) Predictate reports for later physician review with voice recognition technology (Powerscribe; Dictaphone Corporation, Stratford, CT) into a "preliminary" queue accessible only by the practitioner. (3) Provide preliminary oral reports as needed, after consultation 
with a radiologist. This was usually necessary because of a request from a referring physician, an urgent finding, or an important, unexpected finding. (4) Perform these first 3 responsibilities for in-house outpatient cases elsewhere within the ultrasound division when available. (Two practitioners were needed to ensure that 1 was always available to triage the off-site outpatient cases. When their work schedules overlapped and 2 practitioners were available, 1 of them assisted elsewhere in the department, reading outpatient cases performed on-site with staff throughout the day, in much the same way as our residents function. Data about their performance in this role were not kept because they generally functioned less autonomously in this capacity than in their more official role.)

It must be noted that, at this time, our practitioners do not function as sonographers, although they infrequently do some follow-up scanning. In the instances when 2 practitioners are working and 1 is monitoring on-site outpatient cases, this practitioner will sometimes do a scan on an outpatient to solve a problem, in the same way that a staff member, resident, or fellow would do follow-up scanning. The practitioners also do not assist with interventional procedures.

As time went on, we realized that the practitioners could also perform several other subsidiary roles that are not the focus of this report: (1) preparation and presentation of several interesting case conferences for a combined audience of radiologists and sonographers; (2) follow-up of interesting cases to determine outcomes; and (3) performance of quality assurance activities for the ultrasound division.

The practitioners are not allowed to provide final or preliminary reports of any sort unless they have consulted with a staff radiologist. They also do not perform any other function that makes them uncomfortable or uncertain; a radiologist is always available for immediate consultation.

At the end of the clinical day, typically at 5 PM, a radiologist scheduled for the task batch reads the off-site cases in consultation with the practitioner. It is at this time that the dictated report is officially signed and first becomes available to the referring physicians. Detailed records of the time needed were not kept, but the task typically required 1 to 2 hours, with experienced staff members generally requiring less time than junior ones. At program inception, 5 radiologists participated. By the end of the first year, 6 participated, and 8 were participating at the time of manuscript preparation. For the radiology staff, this is now a sought-after activity because an internal "moonlighting" fee is paid for the work.

During every evening batch readout of the offsite cases, the radiologist of the day evaluated the practitioners' performance for each case for the first year. The evaluation was based on the preliminary dictated report because it was believed to be a distillation of all facets of the practitioners' performance. These included ensuring that the sonographic study was complete and that it adequately depicted any pathologic features as well as observations and analyses of the images and recommendations for follow-up ultrasound studies or for additional testing. The report was graded on a 4-part subjective scale, with grammar and syntax errors considered inconsequential, as follows: A, no change in preliminary report/dictation; $\mathrm{B}$, minor change in preliminary report/dictation not affecting patient care (examples: missed small benign renal cyst or missed small gallbladder polyp and unclear or ambiguous wording that if misinterpreted would not substantially affect patient care); C, moderate change in preliminary report/dictation affecting care but not in a dramatic way (examples: missed renal stone or missed gallstone without any other findings to suggest acute cholecystitis, or unclear wording that might substantially affect care if misinterpreted); and D, major change in preliminary report/dictation markedly affecting patient care (example: missed renal tumor or major fetal anomaly).

Records were kept for the first full year that the practitioners functioned, with the exception of the first practitioner, for whom data were not kept for the first 4 weeks. This was because we started the program at approximately the same time we conceived of the idea to publish our results, and we used the first 4 weeks to oversee the beginning of the program and to design this study.

To determine whether the practitioners' performances improved during their first year, their proportions of category $\mathrm{B}$ and $\mathrm{C}$ reports were compared in the following way. The initial proportions of category $\mathrm{B}$ and $\mathrm{C}$ reports were determined for each practitioner by pooling the results of their first 2 months of performance. The final proportions of category $\mathrm{B}$ and $\mathrm{C}$ reports were determined, again for each practitioner, by 
pooling the results of the last 2 months of their first year. For each practitioner, the differences between these initial and final proportions were compared assuming a binomial distribution and by means of the standard error of the difference between proportions. Statistical significance was assumed at the $P<.05$ level.

In addition, a record was kept of the number of patient callbacks because of a study judged to be incomplete at the time of formal interpretation. Records were also kept of complaints from referring nonradiologists about any facet of the program.

\section{Results}

The candidates successfully passed the oral examinations on their first attempt. Practitioner 1 achieved a mean score of 70.8 on the examination, completing it in 14 minutes. Practitioner 2 achieved a mean score of 70.9 , completing the examination in 18 minutes.

The data for evaluation of the practitioners' performances are given in Table 1 and Figure 1. Both practitioners monitored and evaluated the sonographic studies of nearly the same number of patients, 2858 for practitioner 1 and 2825 for practitioner 2, although the data for practitioner 1 only covered a span of 11 months. There were no category D reports for either practitioner. Approximately $96 \%$ of the reports for both practitioners were category A.

For practitioner 1, the initial proportion of category B reports (for the first 2 months) was 0.0787 (28/356); the final proportion of category B reports (for the last 2 months) was 0.0224 (9/402). The proportion of final category B reports was statistically significantly lower than the initial proportion $(P<.001)$. Similarly, the initial proportion of category $\mathrm{C}$ reports (for the first 2 months) was 0.0112 (4/356); the final proportion of category $\mathrm{C}$ reports (for the last 2 months) was $0.00(0 / 402)$. The proportion of final category $C$ reports was not statistically significantly lower than the initial proportion $(P=.06)$.

For practitioner 2, the initial proportion of category B reports (for the first 2 months) was 0.110 (41/374); the final proportion of category B reports (for the last 2 months) was 0.00965 $(5 / 518)$. The proportion of final category B reports was statistically significantly lower than the initial proportion $(P<.0001)$. Similarly, the initial proportion of category $\mathrm{C}$ reports (for the first 2 months) was 0.0107 (4/374); the final proportion of category $\mathrm{C}$ reports (for the last 2 months) was $0.00(0 / 518)$. The proportion of final category $\mathrm{C}$ reports was not statistically significantly lower than the initial proportion $(P=.06)$.

All studies monitored by the practitioners were judged to be acceptable for formal interpretation. There were no patient callbacks for either practitioner in the first year.

Detailed records were not kept regarding the number of times a staff radiologist was consulted by a practitioner during a workday; however, the number of consultations was low enough that the consultations were not viewed as a burden by the staff radiologists, and it is estimated that those interactions usually occurred only once or twice per day.

No complaints about the program from ordering physicians or ancillary personnel associated with those physicians were registered with, or have become known to, the practitioners or the attending radiologists during the entire duration of the program. One staff radiologist disapproved of the program; because participation in the evening readout was voluntary, he chose not to participate.

\section{Discussion}

In our department, as in most other institutions, the current shortage of radiologists has made it difficult to hire and retain academic radiologists. Workloads have increased, and time for academic pursuits has decreased.

In our ultrasound division, this shortage of faculty was acutely exacerbated by the simultaneous opening of several off-site clinics that provide sonography via teleradiology to our in-

Table 1. Practitioner Performance by Examination Subtype: First-Year Data

\begin{tabular}{lcc}
\hline Parameter & Practitioner 1 & Practitioner 2 \\
\hline Patients & 2858 & 2825 \\
Category A reports, \% & 96.2 & 96.1 \\
Category B reports, \% & 3.5 & 3.6 \\
Category C reports, \% & 0.3 & 0.2 \\
Category D reports, \% & 0.0 & 0.0 \\
\hline
\end{tabular}

Category $A$ indicates no change in preliminary report/dictation; $B$, minor change in preliminary report/dictation not affecting patient care; $C$, moderate change in preliminary report/dictation affecting care but not in a dramatic way; and $D$, major change in preliminary report/dictation markedly affecting patient care. 


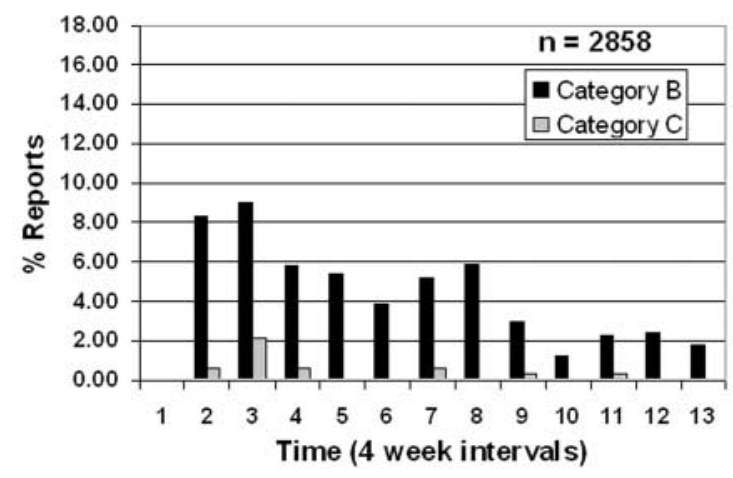

A

Figure 1. Charts showing the performance of each practitioner over the first year as judged by the final evaluations of their dictated reports ( $A$, no change; $B$, minor change not affecting patient care; $C$, moderate change not affecting care in a dramatic way; and $D$, significant change markedly affecting care). There were no category $D$ reports for either practitioner. Category $A$ reports are not indicated because the resultant scale change would dwarf the appearance of the category B and C reports; the percentage of category A reports is $100 \%-(\% B+C$ reports). For each practitioner, there was a statistically significant decrease in the proportion of category B reports by the end of the study compared with the beginning (see "Results"); however, there was not a statistically significant decrease in the proportion of category $C$ reports, likely because of the very low frequency of category $C$ reports at the beginning of the study (see "Results"). A, Results for practitioner 1. Data for the first 4 weeks for practitioner 1 were not collected (as described in "Materials and Methods"). B, Results for practitioner 2.

house PACS. Four scanners went online at the same time, immediately increasing our daily workload of approximately 50 patients per day by an additional 20 to 30 patients per day. It is our custom to review the images before a patient is discharged from the department so that, should they be required, additional images may be obtained to complete an examination. Exacerbating the situation was the need to respond to at least 1 work-disrupting phone call from the off-site sonographer announcing the completion of each case, as well as the need to make a subsequent call to the sonographer to release the patient or to ask for additional images. This made the workload nearly intolerable in our environment, in which considerable time in addition to that required to review the images and generate the reports must be devoted to teaching, interacting with referring physicians, performing ultrasound-assisted procedures, and assisting with sonographic localizations in the operating room. To meet the demands of this abrupt increase in workload, we trained 2 experienced sonographers to triage and predictate these off-site cases in the manner already described.

The success of our program far exceeded our initial expectations. We expected a small but appreciable incidence of substantial errors and were quite surprised that there were none in more than 5000 patients. We also did not expect that $96 \%$ of all studies would result in reports that did not need revision. We were extremely pleased with these results; however, the low prevalence of categories B, C, and D reports should not be taken to mean that the supervising radiologist added little, if any, additional value to the examinations. The practitioners were encouraged to seek the help and input of staff radiologists throughout the day whenever they experienced uncertainty, difficulty, or uneasiness during the evaluation of a case. Data were not kept on the frequency of these contacts, but they usually occurred once or twice daily and sometimes more often than that. If input from a radiologist had not been available for these cases, which were often the more difficult, if not the most difficult, cases, it is likely that the rate of important errors would have been higher. This situation is analogous to that with experienced residents, who are often capable of independently interpreting most ultrasound cases satisfactorily.

We were also surprised to see such similar results for the 2 practitioners (Table 1 and Figure 1). This was by chance, because the radiologists were free to grade the daily performances of each practitioner as they saw fit. Furthermore, ongoing tallies of results were not available to the radiologists.

Acknowledgment should be given to 2 recent technological advances, without which it is unlikely we would have instituted our program: voice recognition transcription and PACS technology with video clips. Without voice recognition transcription, it would have been impossible 
for the reports of all studies to have been available at the batch readout session at the end of the day. The availability of reports and images for simultaneous review was key to allowing batch readout and optimal use of time because the radiologists would not have been comfortable correcting and finalizing reports at a later time after the images had been reviewed. Video clips were also key to the program's success. Our sonographers use them extensively, and although formal data are not kept, many video clips are taken during an examination. Confidence about the completeness of an examination is enhanced when video clips of most areas are provided. Furthermore, it might be considered a limitation that our best sonographers, who were removed from the scanning pool to become practitioners, have to rely on the work of presumably less skilled sonographers for the daily cases. This is where video clip technology is especially important. With the liberal use of video clips, which our practitioners encourage, this potential problem was alleviated. We believe the use of these video clips is an important reason why there were no patient callbacks for an examination judged to be incomplete during our first year.

Historically, retention of our best sonographers has been difficult; internal advancement occurred only when a management position opened, forcing most of those wanting to advance their careers to leave for outside positions. Now that another pathway for advancement is available, we hope that our sonographers will stay to compete for these positions as they become available. We are currently interviewing internal candidates for a third practitioner position.

It is important to stress what our practitioners do not do. They do not issue formal reports for the medical record and do not give any preliminary reports to referring physicians or their staff without the oversight of a radiologist. This would have caused ethical concerns and would have encountered considerable resistance from our radiologists and perhaps from referring physicians. Additionally, the legal implications of such actions are unknown. Since the inception of the program, we have believed that it is extremely important for a radiologist to oversee the activities of the practitioners, and in no way do we advocate that our practitioners be allowed to interpret sonographic studies or issue final reports without the oversight of a radiologist.
It is also important to stress that the roles of our practitioners are not fixed and will be allowed to evolve to best fit our needs. One potential change that is envisioned is the use of our practitioners to assist in training our residents to scan, because our residents currently receive less scanning training than they desire. We are exploring the use of a practitioner in a formal setting to spend 1 day per week with residents scanning patients and showing residents how to scan, supervising the residents' scanning, and also predictating the cases for later review by a staff radiologist. This has the added benefit of allowing our practitioners to retain their scanning skills.

Our experience is unlikely to apply to all practices and likely applies only to those whose policy is for someone else, in addition to the sonographer, to check the examinations for quality and completeness. For those believing that sonographers alone can make the determination, and that sonographic studies can be batchread adequately without input into real-time quality control of the images and without the option to request additional video clips to solve difficult problems and to answer questions raised by the original images, our concept offers little benefit except for the scribing function.

There were several limitations to our study. First, the review of the practitioners' performance was subjective. We could not devise a more quantitative way to assess their performance for the range of ultrasound studies performed. Each radiologist applied the evaluation criteria as consistently as possible, and we believe the results are sufficient to prove our assertions. Second, our study cannot exclude errors of omission. If an examination presented by the practitioner for interpretation did not include an abnormality, the abnormality would also have been missed by the staff radiologist at formal interpretation; however, when the studies were formally reviewed, the interpreting radiologists believed all examinations to be complete, and in no instances were patients called back for additional images. It seems unreasonable to hold the practitioners to a higher standard than that applied to the radiologists. Third, we did not verify that the final diagnoses for all studies were correct. It was considered adequate for the practitioners' impressions to match those of the radiologists.

In summary, it is difficult to imagine incorporating the number of examinations that our practitioners oversee into our daily workload in a less 
burdensome way. As our program is structured, it is crucial to have the oversight of a radiologist available at all times. It is again important to stress that our practitioners do not issue formal, unsupervised reports. Medical action is not taken from their opinions without the oversight of a radiologist, nor do we advocate that they adopt this role in the future. We recommend our setup for any site wishing to ease radiologists' workloads, as long as a qualified sonographer is available to assume the position of an ultrasound practitioner. In the short term, the use of assistants will likely continue to evolve locally in ways best suited to fit local needs. Formal sanctioning and credentialing, if it occurs, may result more from the evolution of these positions than from the attempts of any body to impose its requirements on others.

\section{References}

1. Kiernan B, Rosenbaum HD. The impact of a physician assistant in diagnostic radiology [PA-DR] on the delivery of diagnostic radiologic services. Invest Radiol 1977; 12:7-14.

2. Brealey S, King DG, Crowe MTI, et al. Accident and emergency and general practitioner plain radiograph reporting by radiographers and radiologists: a quasi-randomized controlled trial. Br J Radiol 2003; 76:57-61.

3. Loughran CF. Reporting of fracture radiographs by radiographers: the impact of a training programme. $\mathrm{Br} J$ Radiol 1994: 67:945-950.

4. Robinson PJA. Plain film reporting by radiographers: a feasibility study. Br J Radiol 1996; 69:1171-1174.

5. Lockhart ME, Robbin ML, Berland LL, Smith JK, Canon CL, Stanley RJ. The sonographer practitioner: piece to the radiologist shortage puzzle. J Ultrasound Med 2003; 22:861-864.

6. Benacerraf BR, Bromley BS, Shipp TD, et al. The making of an advanced practice sonographer. J Ultrasound Med 2003; 22:865-867.

7. DuBose TJ, Lockhart ME, Berland LL, Robbin ML. The sonographer practitioner and advanced practice sonographer [letter]. J Ultrasound Med 2003; 22:1420-1422. 